\title{
¿Assimilation of Radial Velocity from Coastal NEXRAD into HWRF for Improved Forecasts of Landfalling Hurricanes
}

\author{
YING WANG ${ }^{\mathrm{a}, \mathrm{b}}$ AND ZHAOXIA PU ${ }^{\mathrm{a}}$ \\ ${ }^{a}$ Department of Atmospheric Sciences, University of Utah, Salt Lake City, Utah
}

(Manuscript received 16 September 2020, in final form 29 December 2020)

\begin{abstract}
The benefits of assimilating Next Generation Weather Radar (NEXRAD) radial velocity data for convective systems have been demonstrated in previous studies. However, impacts of assimilation of such high spatial and temporal resolution observations on hurricane forecasts have not been demonstrated with the National Centers for Environmental Prediction (NCEP) Hurricane Weather and Research Forecasting (HWRF) system. This study investigates impacts of NEXRAD radial velocity data on forecasts of the evolution of landfalling hurricanes with different configurations of data assimilation. The sensitivity of data assimilation results to influencing parameters within the data assimilation system, such as the maximum range of the radar data, superobservations, horizontal and vertical localization correlation length scale, and weight of background error covariances, are examined. Two hurricane cases, Florence and Michael, which occurred in the summer of 2018 are chosen to conduct a series of experiments. Results show that hurricane intensity, asymmetric structure of inland wind and precipitation, and quantitative precipitation forecasting are improved. Suggestions for implementation of operational configurations are provided.
\end{abstract}

KEYWORDS: Hurricanes/typhoons; Numerical weather prediction/forecasting; Data assimilation; Radars/radar observations

\section{Introduction}

The Next Generation Weather Radar (NEXRAD) system is composed of 160 Weather Surveillance Radar-1988 Doppler (WSR-88D) operational weather radar sites throughout the United States and has proven to be a great resource in monitoring severe convective systems (e.g., Laroche and Lang 2017; Ansari et al. 2018; Hu et al. 2020). WSR-88D weather radar observations have a high temporal and spatial resolution, which provides a rich data source for assimilation into numerical weather prediction (NWP) models (e.g., Sun and Crook 1997; Snyder and Zhang 2003; Alpert and Kumar 2007; Lai et al. 2019). Previous studies (Sun 2005; Jung et al. 2010; Posselt et al. 2015) have demonstrated that the assimilation of Doppler radar data shows positive effects for convective cell forecasts at convectivepermitting scales. The improvements gained in influencing structural fields could further improve forecasts of convection evolution and precipitation (e.g., Xiao et al. 2005; Tong and Xue 2005; Gao and Stensrud 2012). Besides, NEXRAD observations could also be utilized for coastal high-impact weather (e.g., tropical cyclones) to improve the prediction of the central location, intensity, and associated precipitation of tropical cyclones near the landfall (Zhao and Jin 2008; Rappaport et al. 2009).

In operational data assimilation systems, such as the National Centers for Environmental Prediction (NCEP)'s Gridpoint

D Denotes content that is immediately available upon publication as open access.

\footnotetext{
${ }^{\mathrm{b}}$ Current affiliation: School of Marine Sciences, Nanjing University of Information Science and Technology, Nanjing, China.
}

Corresponding author: Prof. Zhaoxia Pu, zhaoxia.pu@utah.edu
Statistical Interpolation (GSI) system, the configurations for radar radial wind assimilation have been explored to better leverage such high-resolution data to improve model forecasts. For instance, Lippi et al. (2019) discussed a refinement of radial wind superobservation processing in GSI and applied it to a tornado case. They considered the smoothing magnitude and elevation angle range for radar radial wind assimilation in an operational model. However, until recently, the ground-based NEXRAD data have not been operationally assimilated in the NCEP Hurricane Weather and Research Forecasting Model (HWRF), which has been implemented as an operational model since 2007. Meanwhile, assimilation of airborne TDR radar radial wind data achieved significant improvements in hurricane track and intensity forecasts (Pu et al. 2009; Zhang et al. 2012; Li et al. 2014).

The effective assimilation of NEXRAD data in an operational platform relies on a rapidly updated quality control system for NEXRAD observations. With the polarimetric upgrade of the WSR-88D network, new observations (i.e., differential reflectivity, differential phase, specific differential phase, and correlation coefficient) that better reveal the distribution of hydrometeors inside clouds have become available. Nevertheless, it has been challenging to develop effective quality control methods for the NEXRAD observations. Tang et al. (2014) proposed and implemented a simple $\rho_{\mathrm{HV}}$-based $P-N P$ segregation (dpQC) algorithm, which is currently applied in the real-time national Multi-Radar Multi-Sensor (MRMS) quantitative precipitation estimation (QPE) system. This automated algorithm utilizes polarimetric data (i.e., reflectivity and correlation coefficients) from 180 operational radar sites and environmental data (i.e., temperature) to perform radar quality control with a set of physically based rules (Zhang et al. 2011; Tang et al. 2014). The initial operational capability of MRMS has been verified. Results demonstrated that the dpQC 
TABLE 1. Configurations of data assimilation experiments. In the experiments' names, F stands for experiments for Hurricane Florence and $\mathrm{M}$ denotes experiments for Michael.

\begin{tabular}{|c|c|c|c|c|c|c|}
\hline Expt & $\begin{array}{l}\text { Max radial } \\
\text { range }(\mathrm{km})\end{array}$ & $\begin{array}{c}\text { Radial } \\
\text { range }(\mathrm{km})\end{array}$ & $\begin{array}{l}\text { Horizontal localization } \\
\text { correlation length }(\mathrm{km})\end{array}$ & $\begin{array}{l}\text { Vertical localization correlation } \\
\text { length (grid) }\end{array}$ & $\beta_{1}$ & $\beta_{2}$ \\
\hline $\begin{array}{l}\text { Exp. FCONV; } \\
\text { Exp. MCONV }\end{array}$ & - & - & 300 & $0.2(\ln p)$ & 0.2 & 0.8 \\
\hline Exp. F01; Exp. M01 & 100 & 9 & 300 & $0.2(\ln p)$ & 0.2 & 0.8 \\
\hline Exp. F02; Еxp. M02 & 150 & 9 & 300 & $0.2(\ln p)$ & 0.2 & 0.8 \\
\hline Exp. F03; Exp. M03 & 200 & 9 & 300 & $0.2(\ln p)$ & 0.2 & 0.8 \\
\hline Exp. F04; Exp. M04 & 200 & 3 & 300 & $0.2(\ln p)$ & 0.2 & 0.8 \\
\hline Exp. F05; Exp. M05 & 200 & 3 & 400 & 10 & 0.2 & 0.8 \\
\hline Exp. F06; Exp. M06 & 200 & 3 & 400 & 10 & 0.3 & 0.7 \\
\hline Exp. F07; Exp. M07 & 200 & 3 & 400 & 10 & 0.4 & 0.6 \\
\hline
\end{tabular}

algorithm could remove nonhydrometeor echoes (e.g., wind farms, anomalous propagation ground clutter, and deep biological echoes) at a high computational efficiency (Zhang et al. 2016).

The effectiveness of the dpQC algorithm enables us to assimilate NEXRAD data operationally. In this study, we investigate the optimal operational configuration of the GSI data assimilation system for NEXRAD radial wind assimilation in HWRF based on the quality-controlled data using the dpQC algorithm. Our purpose is to adjust the GSI-based three-dimensional hybrid ensemble-variational (hybrid 3DEnVar, Wang 2010) data assimilation to a configuration suitable for assimilating NEXRAD data. We will emphasize adjusting the maximum radial range, radial range for superobservation boxes, horizontal and vertical localization correlation length, and weights applied to the static background error covariance and ensemble covariance. Two hurricane cases in 2018 are selected to demonstrate the impacts of assimilating radial wind data on hurricane landfall. The model, data, and experimental design are described in section 2. Results and discussion are
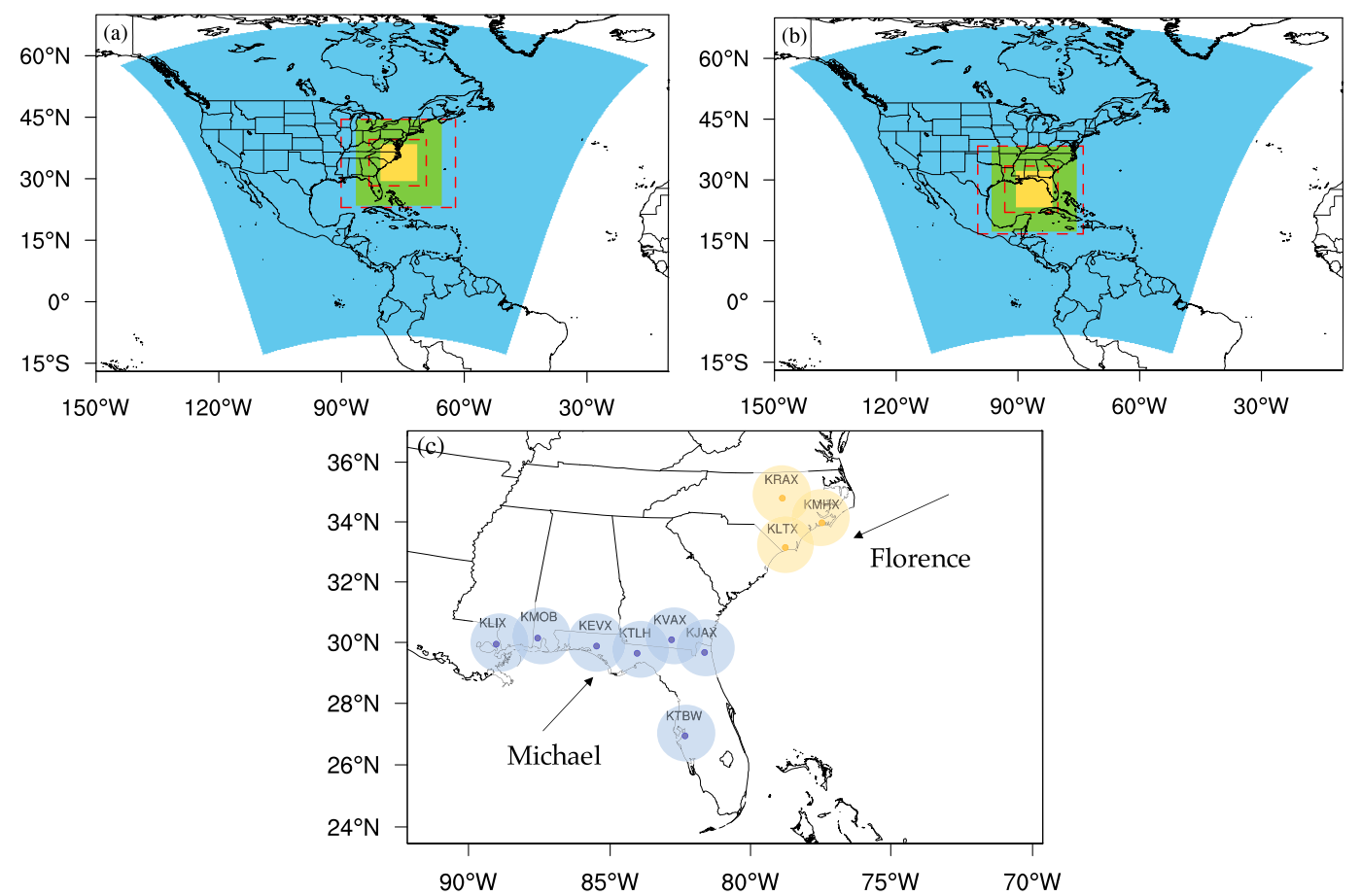

FIG. 1. The domains utilized in HWRF for Hurricanes (a) Florence and (b) Michael. The blue region is the outer domain at 13.5-km horizontal grid spacing. The green and yellow regions are vortex-following domains at $4.5-$ and $1.5-\mathrm{km}$ horizontal grid spacing. The red dashed lines are the ghost domains for $\mathrm{d} 02$ and $\mathrm{d} 03$. (c) The locations of NEXRAD sites employed for data assimilation experiments for Hurricanes Florence and Michael. Data from the KRAX, KLTX, and KMHX sites are used for Hurricane Florence (yellow circled regions); and data from the KLIX, KMOB, KEVX, KTLH, KVAX, KJAX, and KTBW sites are used for Hurricane Michael (blue circled regions). The colored circles show the scanning coverage of each radar site. 

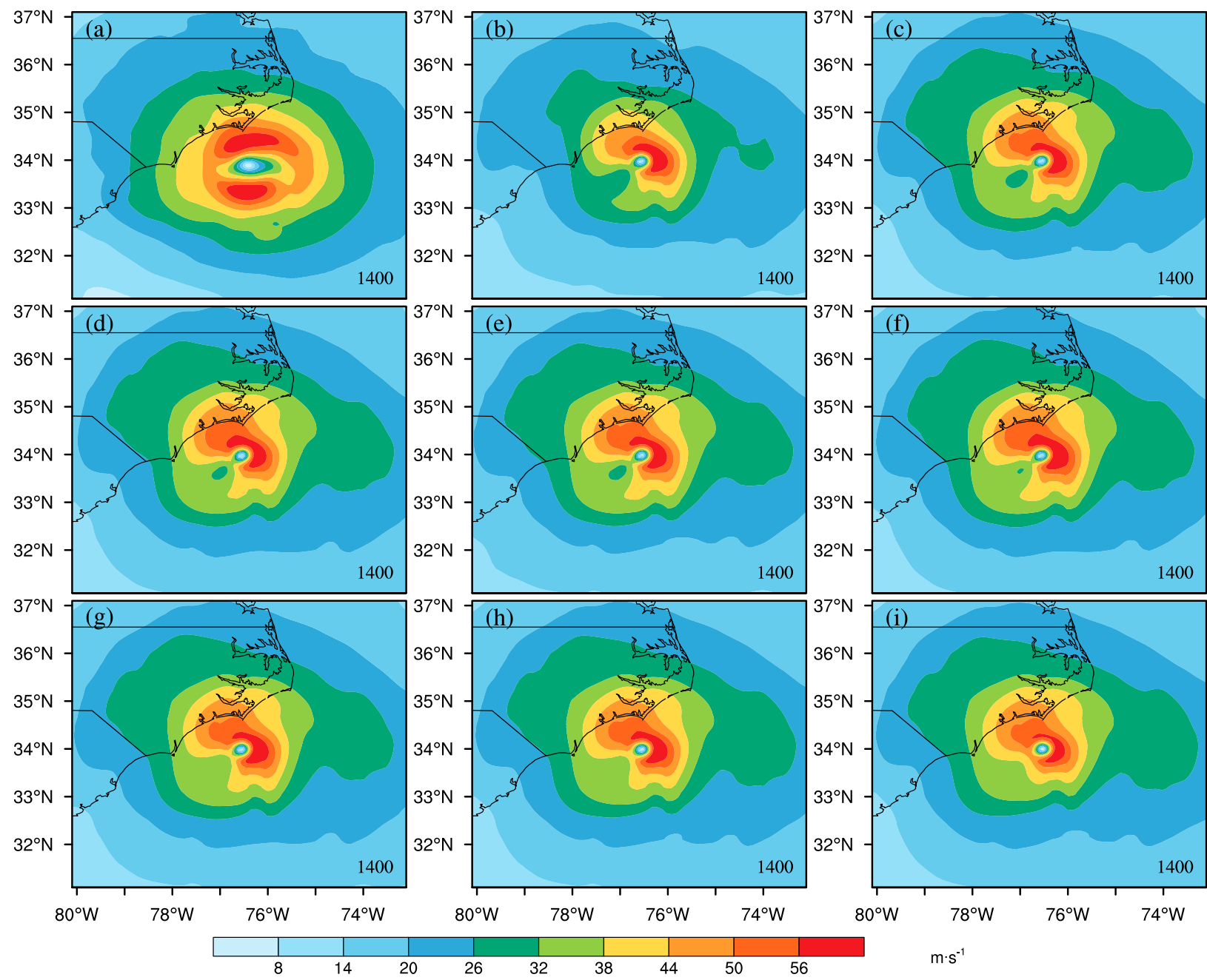

FIG. 2. (a) HRRR horizontal wind field at $925 \mathrm{hPa}$ and horizontal wind analysis field ( $\mathrm{m} \mathrm{s}^{-1}$ ) at the 17th eta level (approximately $925 \mathrm{hPa}$ ) for Florence at 0000 UTC. Corresponding fields from (b) Exp. FCONV, (c) Exp. F01, (d) Exp. F02, (e) Exp. F03, (f) Exp. F04, (g) Exp. F05, (h) Exp. F06, and (i) Exp. F07.

presented in sections 3 and 4. Section 5 offers a summary of this work.

\section{HWRF model, data, and experimental design}

All experiments were conducted with HWRF, version 4.0a (Biswas et al. 2018), which is close to the current operational HWRF at the National Oceanic and Atmospheric Administration's (NOAA) National Centers for Environmental Prediction (NCEP). The HWRF system is composed of the Weather Research and Forecasting (WRF) nonhydrostatic mesoscale model on the E grid dynamic core (Janjić et al. 2010), the Message Passing Interface Princeton Ocean Model for Tropical Cyclones (MPIPOM-TC) (Yablonsky et al. 2015), the NCEP coupler, and the GSI assimilation platform ( $\mathrm{Hu}$ et al. 2018). It is operationally launched every $6 \mathrm{~h}$ in a configuration with a parent domain and two moving nested domains, of which the resolutions are $13.5,4.5$, and $1.5 \mathrm{~km}$, respectively
(Biswas et al. 2018). The physical parameterizations employed in HWRF are the Ferrier (new Eta) microphysics scheme (Ferrier et al. 2002), the Rapid Radiative Transfer Model for GCMs (RRTMG) longwave and shortwave radiation schemes (Iacono et al. 2008), the Unified Noah land surface model (Chen and Dudhia 2001; Mitchell 2005), the hybrid Eddy Diffusivity Mass-Flux (EDMF) Global Forecast System (GFS) scheme (Han et al. 2016), and the scale-aware GFS Simplified Arakawa-Schubert (SASAS) convection scheme (Pan and Wu 1995; Hong and Pan 1998; Pan 2003; Han and Pan 2011).

Two hurricane cases selected for this study are Florence and Michael, which occurred over the northwest Atlantic Ocean in September and October 2018 with a western and a northeastern landfall track, respectively. Assimilation data include conventional data from NCEP's Automated Data Processing (ADP), and NEXRAD radial wind data that have been processed through dpQC quality control in the MRMS system. 


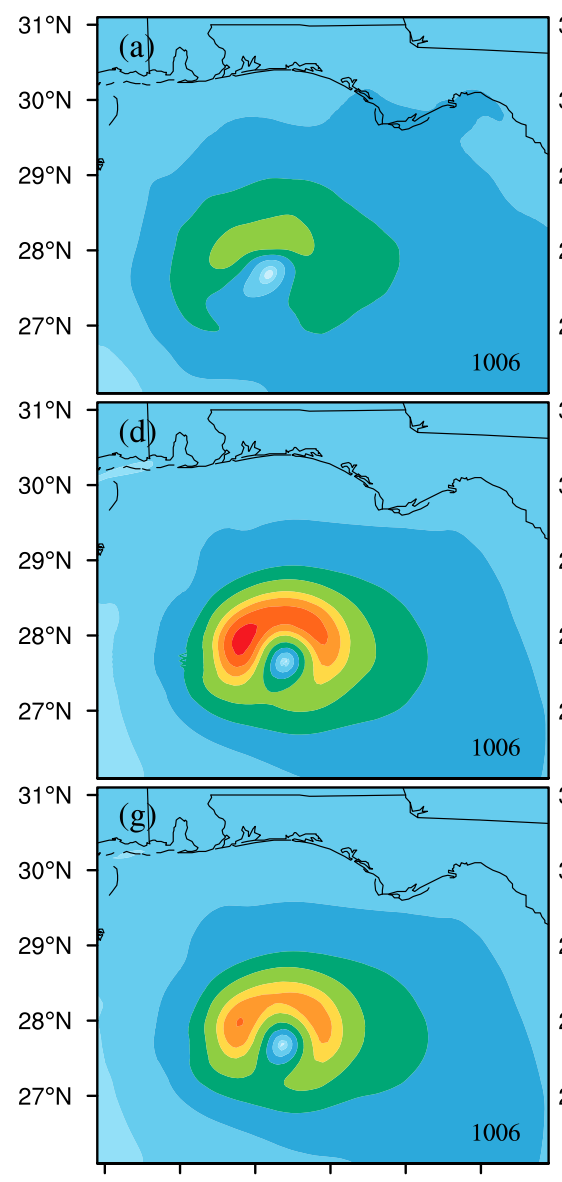

$89^{\circ} \mathrm{W} 88^{\circ} \mathrm{W} 87^{\circ} \mathrm{W} 86^{\circ} \mathrm{W} 85^{\circ} \mathrm{W} 84^{\circ} \mathrm{W}$

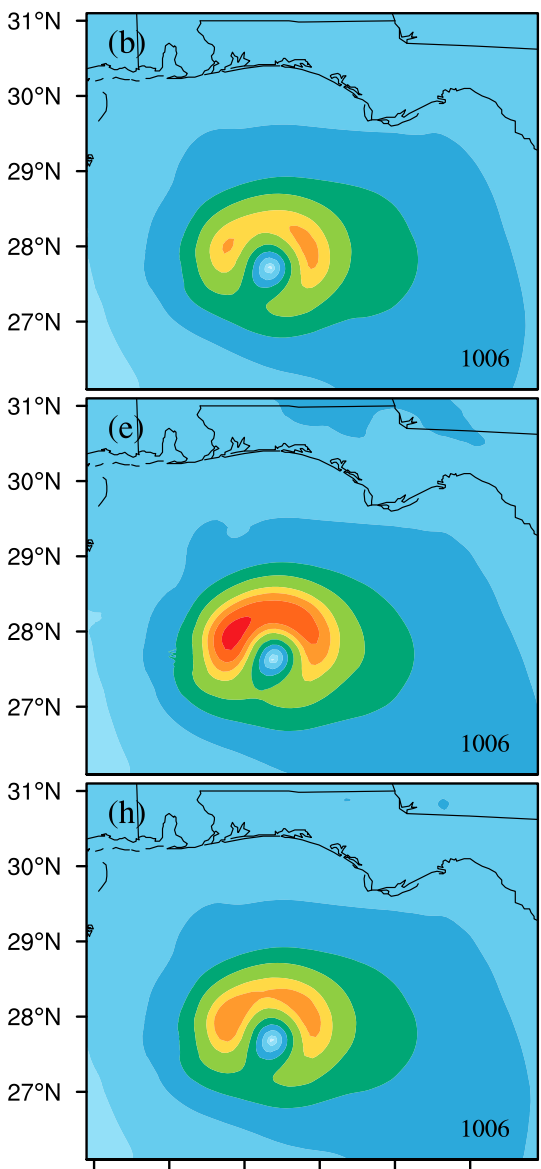

$89^{\circ} \mathrm{W} 88^{\circ} \mathrm{W} 87^{\circ} \mathrm{W} 86^{\circ} \mathrm{W} 85^{\circ} \mathrm{W} 84^{\circ} \mathrm{W}$

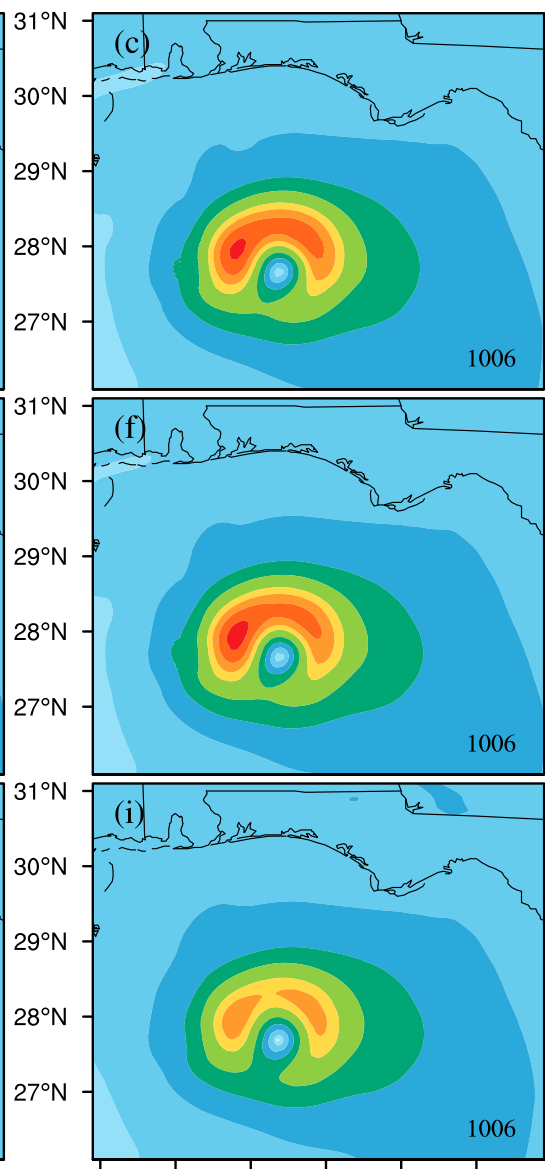

$89^{\circ} \mathrm{W} 88^{\circ} \mathrm{W} 87^{\circ} \mathrm{W} 86^{\circ} \mathrm{W} 85^{\circ} \mathrm{W} 84^{\circ} \mathrm{W}$ $\mathrm{m} \cdot \mathrm{s}^{-1}$

FIG. 3. (a) HRRR horizontal wind field at $925 \mathrm{hPa}$ and horizontal wind analysis field $\left(\mathrm{m} \mathrm{s}^{-1}\right.$ ) at the 17 th eta level (approximately $925 \mathrm{hPa}$ ) for Michael at 0600 UTC 10 Oct 2018. Corresponding wind fields from (b) Exp. MCONV, (c) Exp. M01, (d) Exp. M02, (e) Exp. M03, (f) Exp. M04, (g) Exp. M05, (h) Exp. M06, and (i) Exp. M07.

The dpQC algorithm is a physically based scheme that relies on general and explicit meteorological principles. It contains a basic $\rho_{\mathrm{HV}}$ filter to separate $P$ (precipitation, high $\rho_{\mathrm{HV}}$ ) and NP (nonprecipitation, low $\rho_{\mathrm{HV}}$ ) regions. A 3D reflectivity structure obtained from operational dual-polarization radar stations, together with meteorological environmental data, is utilized to preserve hail, nonuniform beam filling, and melting layer regions in this $\rho_{\mathrm{HV}}$ filter. Additionally, random nonprecipitation pixels that exhibit high $\rho_{\mathrm{HV}}$ values are eliminated by spatial filters and vertical and horizontal consistency checks at a high computational efficiency (Tang et al. 2014).

GSI-based hybrid 3DEnVar data assimilation is employed to conduct conventional and NEXRAD data assimilation for these two hurricane cases. The background error covariance of hybrid 3DEnVar is composed of a flow-dependent background error covariance achieved by 80 GFS ensemble members, and a static background error covariance obtained through the National Meteorological Center (NMC) method. The cost function is as follows (Wang 2010; Biswas et al. 2018):

$$
\begin{aligned}
J\left(\mathbf{x}_{1}^{\prime}, \boldsymbol{\alpha}\right)= & \beta_{1}\left(\mathbf{x}_{1}^{\prime}\right)^{\mathrm{T}} \mathbf{B}_{1}^{-1}\left(\mathbf{x}_{1}^{\prime}\right)+\beta_{2}(\boldsymbol{\alpha})^{\mathrm{T}} \mathbf{A}^{-1}(\boldsymbol{\alpha}) \\
& +\left(\mathbf{y}^{0^{\prime}}-H \mathbf{x}^{\prime}\right)^{\mathrm{T}} \mathbf{R}^{-1}\left(\mathbf{y}^{0^{\prime}}-H \mathbf{x}^{\prime}\right)+J_{c}
\end{aligned}
$$

where $\mathbf{B}_{1}$ is the static background error covariance matrix; $\beta_{1}$ and $\beta_{2}$ are, respectively, the weight applied to the static background error covariance and the ensemble covariance; $\boldsymbol{\alpha}$ contains the extended control variables for ensemble members; $\mathbf{A}$ defines the spatial correlation of $\boldsymbol{\alpha} ; \mathbf{y}$ is the innovation vector; $H$ is the observation operator; $\mathbf{R}$ is the observational and representativeness error covariance matrix; and $J_{c}$ is a constraint term.

In operational systems, $\beta_{1}$ and $\beta_{2}$ are set to 0.2 and 0.8 , which offers more weight to the flow-dependent background error covariance. To explore the influence of different weights on NEXRAD radial wind assimilation, experiments will be conducted in this study using different combinations of $\beta_{1}$ and $\beta_{2}$ (See Table 1, also next section). The forecast hurricane track is quite sensitive to different combinations. Besides the adjustable $\beta_{1}$ and $\beta_{2}$ in the assimilation algorithm, horizontal and 

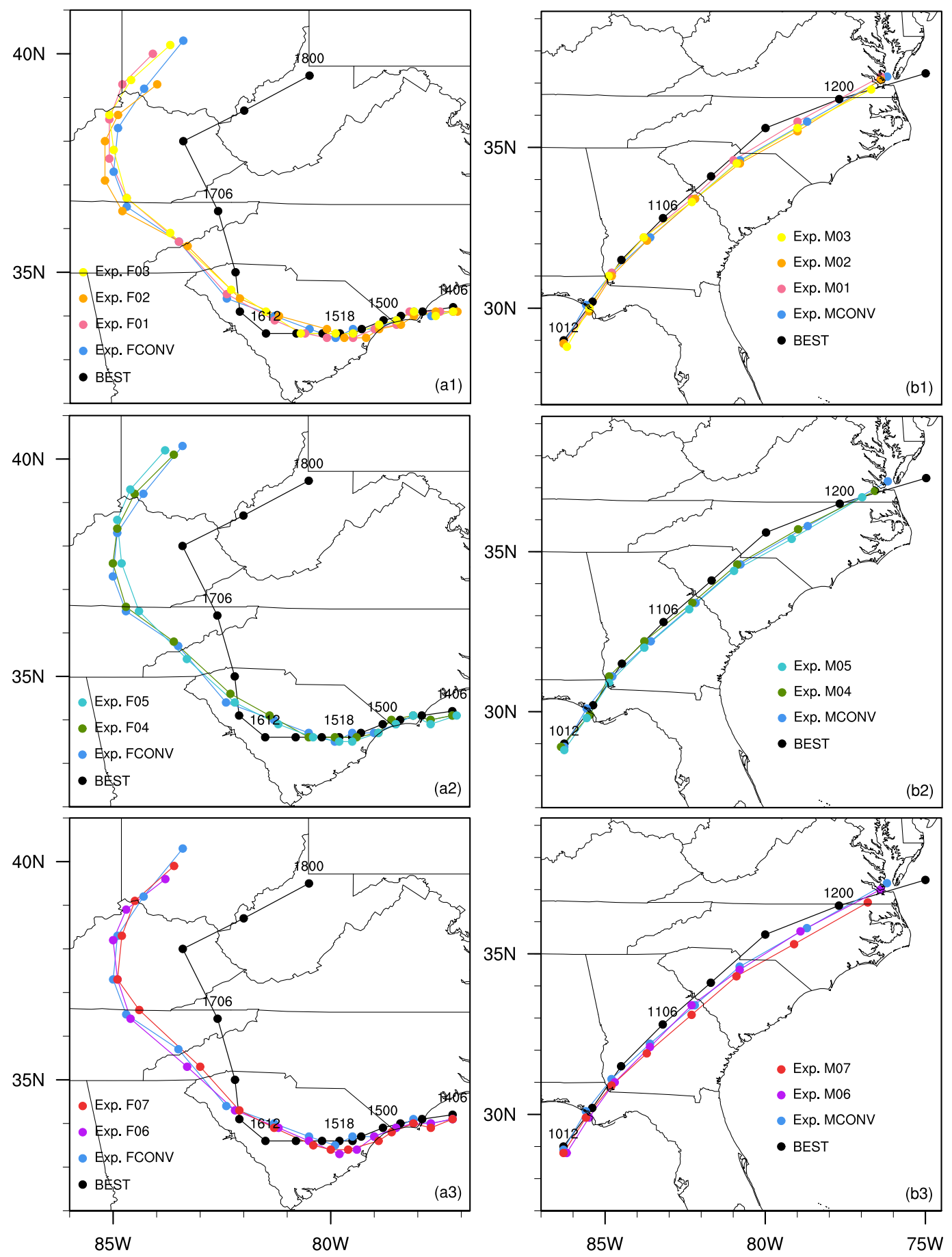

FIG. 4. Forecast track of conventional and NEXRAD assimilation experiments for Florence and Michael. (a) Florence (0600 UTC 14 Sep-0000 UTC 18 Sep 2018) and (b) Michael (1200 UTC 10 Oct-0000 UTC 12 Oct 2018).

vertical localization correlation length, radial range for superobservation boxes, and maximum radial range are adaptable for NEXRAD radial wind assimilation in GSI. To investigate an appropriate configuration for radial wind assimilation and evaluate its impact on hurricane landfall, conventional assimilation experiments (assimilate NCEP ADP data) and seven NEXRAD assimilation experiments (assimilate both NCEP ADP and NEXRAD radial velocity) are designed for Hurricanes Florence and Michael. The conventional assimilation experiments are named 

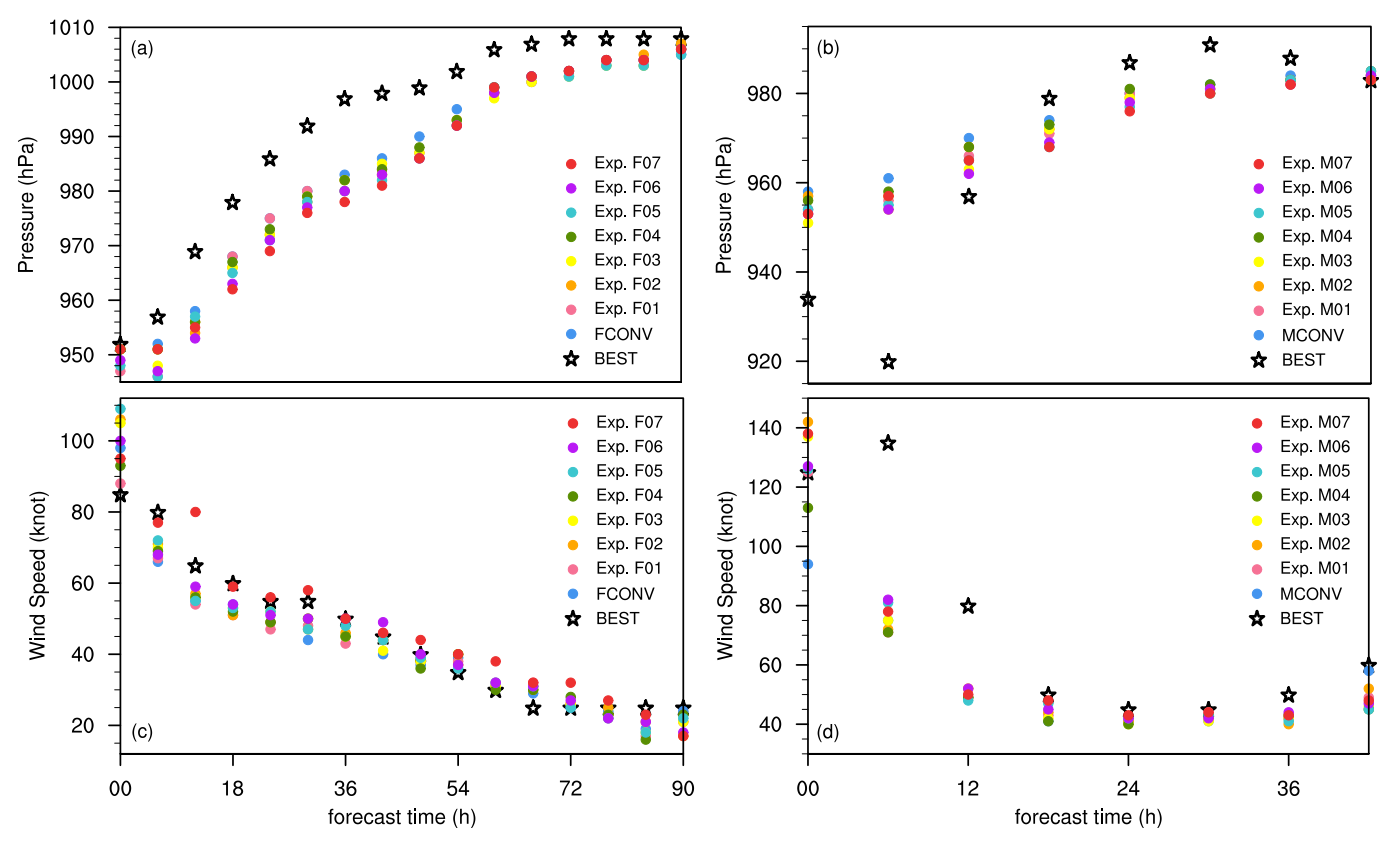

FIG. 5. Forecast hurricane intensity for all experiments. (a) The minimum pressure (hPa) and (c) the maximum wind $\left[\mathrm{kt} ; 1 \mathrm{kt} \approx 0.51 \mathrm{~m} \mathrm{~s}^{-1}\right.$ ) for Florence (0600 UTC 14 Sep-0000 UTC 18 Sep 2018). (b) The minimum pressure and (d) the maximum wind for Michael (1200 UTC 10 Oct-0000 UTC 12 Oct 2018). The $x$ axis is the forecast time (h).

Exp. FCONV and Exp. MCONV, respectively, for Florence and Michael.

All experiments with NEXRAD radial wind assimilation are the same as experiments that assimilate conventional observations, but the assimilation of radial wind data has different configurations (see Table 1). In the operational HWRF configuration, a blending option is employed to eliminate data assimilation increments within $150 \mathrm{~km}$ of the storm center and gradually reintroduce these increments between 150 and $300 \mathrm{~km}$ (Biswas et al. 2018). However, this blending scheme could produce an unrealistic vortex structure in the lower- to midtropospheric inner-core region (Zhang and $\mathrm{Pu}$ 2019). To evaluate the impacts of assimilating NEXRAD radial wind on the hurricane's inner core, the blending option is turned off and only vortex relocation is employed in the vortex initialization procedure. For Florence, the coastal NEXRAD sites Raleigh/Durham, North Carolina (KRAX), Wilmington, North Carolina (KLTX), and Morehead City, North Carolina (KMHX), are utilized to obtain radial wind data. For Michael, the seven NEXRAD sites New Orleans, Louisiana (KLIX), Mobile, Alabama (KMOB), Eglin Air Force Base, Florida (KEVX), Tallahassee, Florida (KTLH), Moody Air Force Base, Georgia (KVAX), Jacksonville, Florida (KJAX), and Tampa, Florida (KTBW), are employed (Fig. 1). Radial wind data from all elevation angles are assimilated with an assimilation window ranging from -0.5 to $0.5 \mathrm{~h}$ based on the analysis time. The landfall time for Florence is 1115 UTC 14 September 2018, and that for Michael is 1730 UTC 10 October 2018. Due to the limitation of coastal NEXRAD coverage, all experiments are started $24 \mathrm{~h}$ before landfall time with cycled data assimilated using conventional data at a 6-h interval until the time when NEXRAD data assimilated and this time period is considered as a spinup period. Then Exp. FCONV and Exp. MCONV continued with the assimilation of conventional data (i.e., NCEP ADP), while the NEXRAD assimilation experiments (all other experiments in Table 1 except for Exp. FCONV and Exp. MCONV) assimilate both NCEP ADP and NEXRAD data. For Florence, the NEXRAD assimilation experiments were cycling conducted at 0000 and 0600 UTC 14 September 2018, and for Michael were conducted at 0600 and 1200 UTC 10 October 2018. A 126-h forecast was made after each experiment. Results were evaluated for the period which has NHC best track data available.

\section{Experimental results}

To evaluate the factors that could influence NEXRAD radial velocity data assimilation, a series of numerical experiments are conducted with different configurations (Table 1). The factors with significant influence on NEXRAD data assimilation are identified. A possible best choice for potential operational applications will be recommended.

\section{a. Maximum radial range}

Maximum radial range determines the horizontal coverage of radial velocity data and is utilized to construct superobservations. A good average and a minimum dependency between adjacent superobservations are usually ensured by a large enough area, which is determined by maximum radial range (Alpert and Kumar 2007). For continental convectivescale data assimilation with GSI, the default maximum radial range is set to $100 \mathrm{~km}$ for single NEXRAD stations, but this is not sufficient for hurricane cases because hurricanes can expand to several hundred kilometers. To obtain better radial 


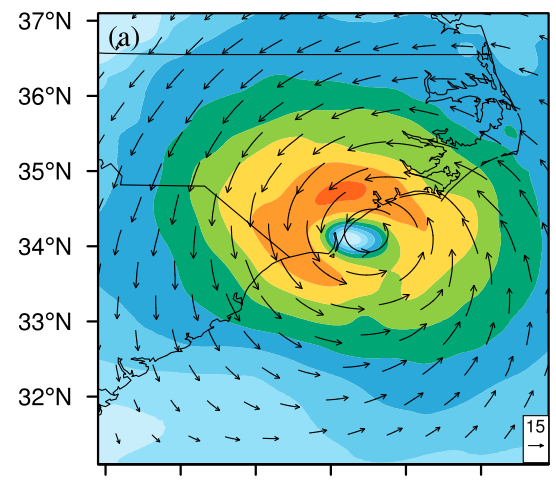

$81^{\circ} \mathrm{W} 80^{\circ} \mathrm{W} 79^{\circ} \mathrm{W} 78^{\circ} \mathrm{W} 77^{\circ} \mathrm{W} 76^{\circ} \mathrm{W}$

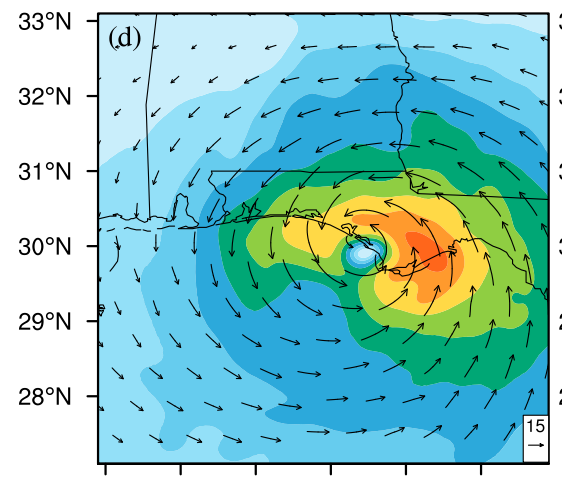

$89^{\circ} \mathrm{W} 88^{\circ} \mathrm{W} 87^{\circ} \mathrm{W} 86^{\circ} \mathrm{W} 85^{\circ} \mathrm{W} 84^{\circ} \mathrm{W}$

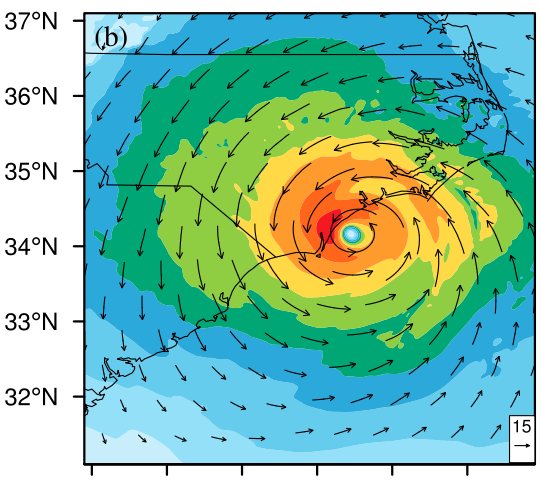

$81^{\circ} \mathrm{W} 80^{\circ} \mathrm{W} 79^{\circ} \mathrm{W} 78^{\circ} \mathrm{W} 77^{\circ} \mathrm{W} 76^{\circ} \mathrm{W}$

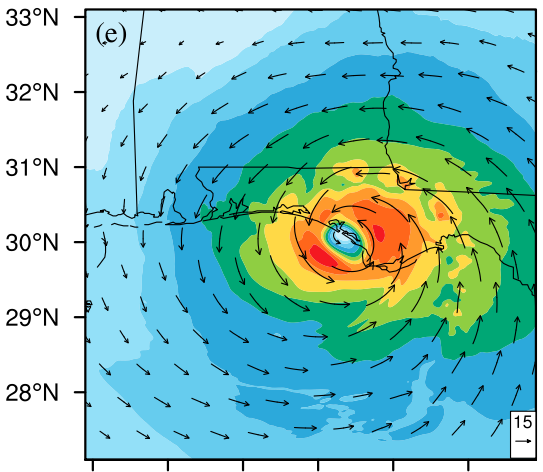

$89^{\circ} \mathrm{W} 88^{\circ} \mathrm{W} 87^{\circ} \mathrm{W} 86^{\circ} \mathrm{W} 85^{\circ} \mathrm{W} 84^{\circ} \mathrm{W}$

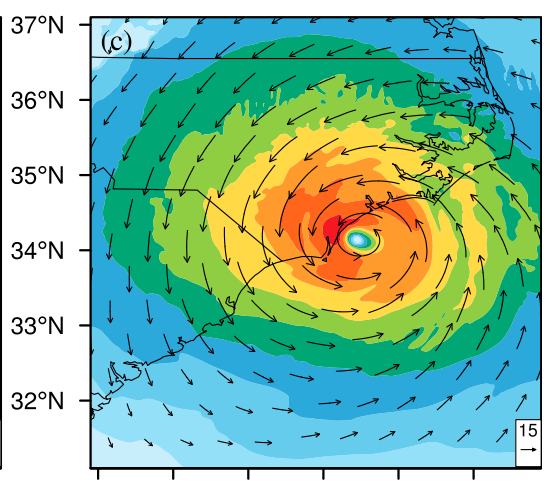

$81^{\circ} \mathrm{W} 80^{\circ} \mathrm{W} 79^{\circ} \mathrm{W} 78^{\circ} \mathrm{W} 77^{\circ} \mathrm{W} 76^{\circ} \mathrm{W}$

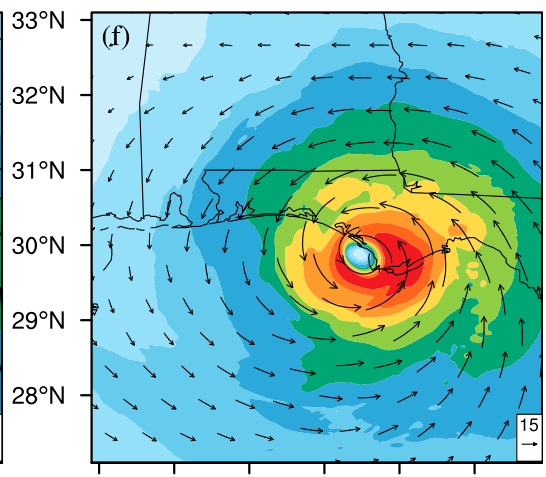

$89^{\circ} \mathrm{W} 88^{\circ} \mathrm{W} 87^{\circ} \mathrm{W} 86^{\circ} \mathrm{W} 85^{\circ} \mathrm{W} 84^{\circ} \mathrm{W}$

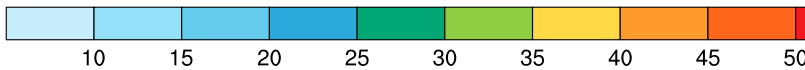

$\mathrm{m} \cdot \mathrm{s}^{-1}$

FIG. 6. Horizontal wind $\left(\mathrm{m} \mathrm{s}^{-1}\right)$ at $925 \mathrm{hPa}$ for Florence (1200 UTC 14 Sep 2018) and Michael (1800 UTC 10 Oct 2018$)$ at landfall. Colored contours represent wind speed, and vectors represent wind direction. The wind fields of HRRR for (a) Florence and (d) Michael. The forecasts of (b) Exp. FCONV and (c) Exp. F06. The forecasts of (e) Exp. MCONV and (f) Exp. M06.

velocity data coverage near the coast for hurricanes, a larger maximum radial range is tested. Exp. F01-03 and Exp. M0103, with a maximum radial range ranging from 100 to $200 \mathrm{~km}$, are aimed at investigating the sensitivity of maximum radial range. To evaluate the reasonability of different configurations for assimilating NEXRAD radial velocity, Figs. 2 and 3 illustrate analysis fields of horizontal winds at the 17 th eta level (approximately $925 \mathrm{hPa}$ ) against NOAA/ESRL High-Resolution Rapid Refresh (HRRR) high-resolution $(\sim 3 \mathrm{~km})$ operational analysis. HRRR analysis is the finest-resolution operational regional analysis available, and it provides reliable analysis for hurricane cases (Bytheway et al. 2017; Yue and Gebremichael 2020). Figures 2c-e and 3c-e in these two figures represent results of Exp. F01-03 and Exp. M01-03 for these two hurricane cases. For Florence, assimilating radar radial velocity has a positive impact on adjusting the radius of horizontal wind greater than $26 \mathrm{~ms}^{-1}$. Compared with Exp. FCONV, Exp. F01-03 presents a looser pattern, as in HRRR. With the increase of maximum radial range ranging from 100 to $200 \mathrm{~km}$, the underestimated southwestern wind region (greater than $32 \mathrm{~m} \mathrm{~s}^{-1}$ ) of the vortex shows positive improvements. For Michael, there is an underestimation of maximum wind speed in HRRR, when referring to the hurricane report of maximum wind speed of about $61.7 \mathrm{~m} \mathrm{~s}^{-1}$. Assimilating radar radial velocity significantly increases the maximum wind speed and adjusts the maximum region to the northwest, similar to HRRR.

\section{b. Superobservations}

The radial range for superobservation boxes is another essential parameter in superobservation construction. It determines each box size along a radius to reduce the volume of observations (Lippi et al. 2019). Exp. F03-04 and Exp. M03-04 are designed to explore the sensitivity of radial range. The default range in GSI is $9 \mathrm{~km}$ (Exp. F03 and M03). To make high-density superobservations that are more consistent with the HWRF model resolution, we further adjust this range to a lower value of $3 \mathrm{~km}$ (Exp. F04 and M04). For Florence, the underestimated southwestern wind region in Exp. F04 gains a further revision. For Michael, although Exp. M03 achieves the proper maximum wind location in the northwest, Exp. M04 additionally decreases the overestimation of northwestern maximum wind speed.

\section{c. Horizontal and vertical localization correlation length scale}

Compared with conventional data, NEXRAD radial velocity data have a higher spatial resolution. Thus, horizontal and vertical localization correlation lengths, which are ubiquitous 

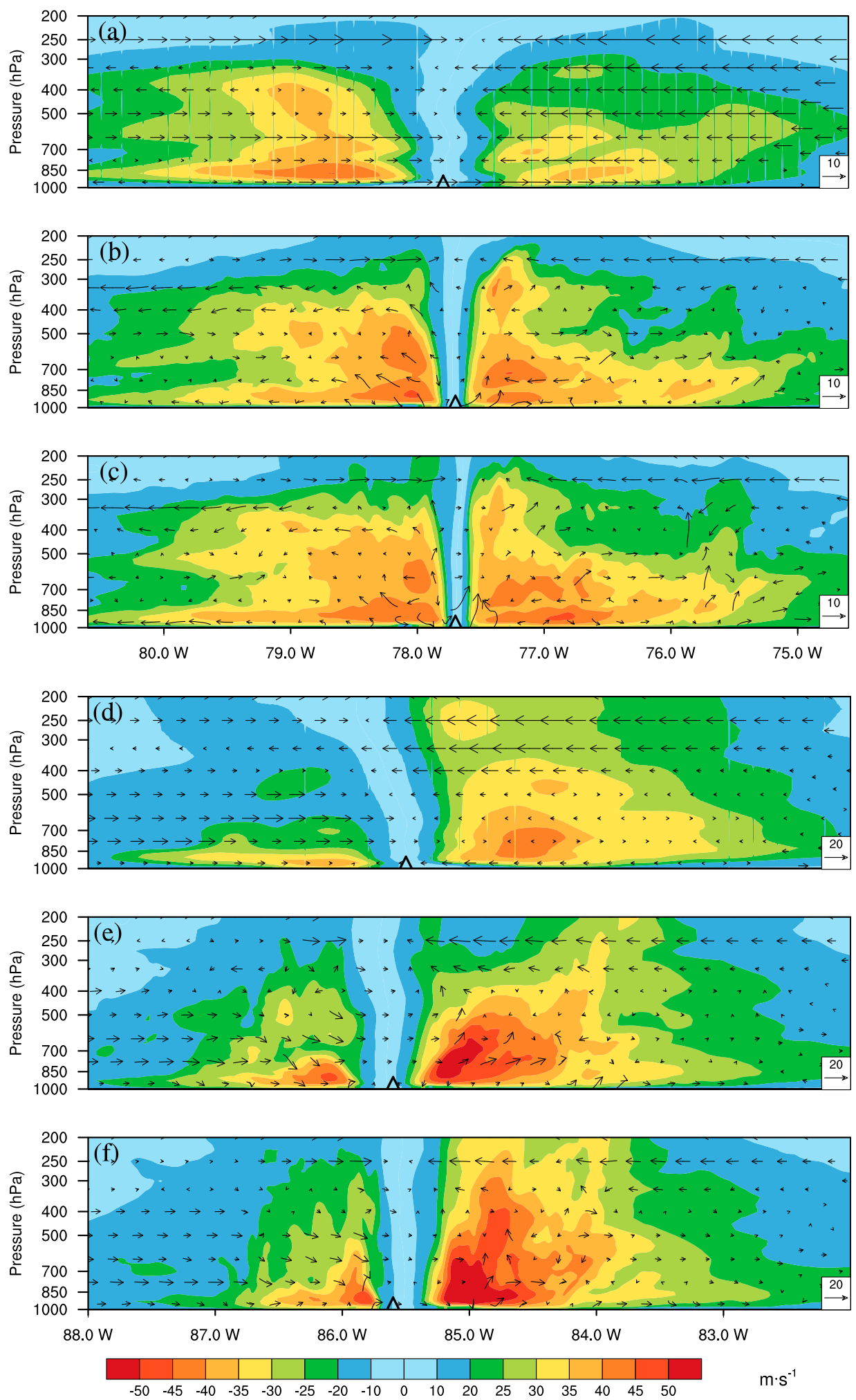

FIG. 7. Wind field of vertical cross section for Florence (1200 UTC 14 Sep 2018) and Michael (1800 UTC 10 Oct 2018) at landfall. Colored contours are tangential wind $\left(\mathrm{m} \mathrm{s}^{-1}\right)$, and vectors denote radial wind $\left(\mathrm{m} \mathrm{s}^{-1}\right)$ and vertical velocity $\left(0.1 \mathrm{~m} \mathrm{~s}^{-1}\right)$. The wind fields of HRRR for (a) Florence and (d) Michael. The forecasts of (b) Exp. FCONV and (c) Exp. F06. The forecasts of (e) Exp. MCONV and (f) Exp. M06. The triangle represents the location of the hurricane eye. 


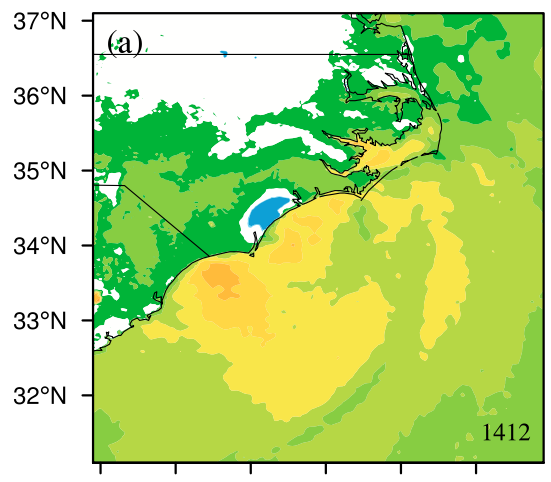

$80^{\circ} \mathrm{W} 79^{\circ} \mathrm{W} 78^{\circ} \mathrm{W} 77^{\circ} \mathrm{W} 76^{\circ} \mathrm{W} 75^{\circ} \mathrm{W}$

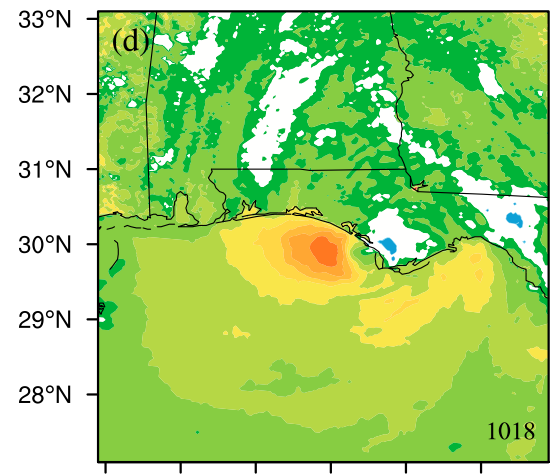

$89^{\circ} \mathrm{W} 88^{\circ} \mathrm{W} 87^{\circ} \mathrm{W} 86^{\circ} \mathrm{W} 85^{\circ} \mathrm{W} 84^{\circ} \mathrm{W}$

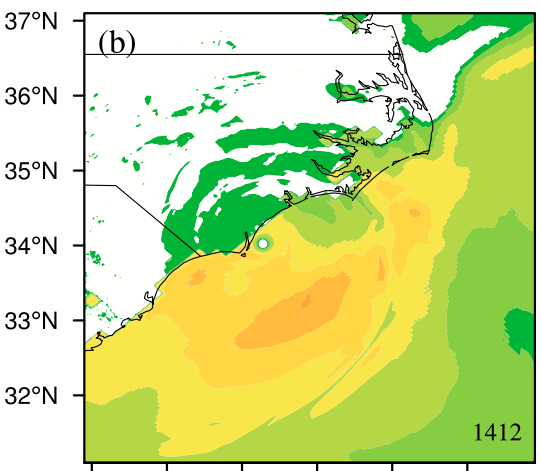

$80^{\circ} \mathrm{W} 79^{\circ} \mathrm{W} 78^{\circ} \mathrm{W} 77^{\circ} \mathrm{W} 76^{\circ} \mathrm{W} 75^{\circ} \mathrm{W}$

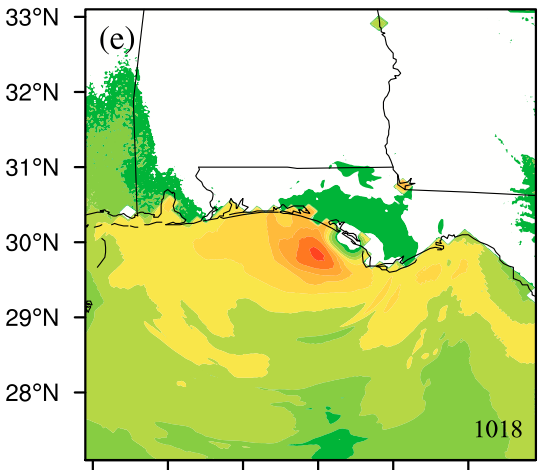

$89^{\circ} \mathrm{W} 88^{\circ} \mathrm{W} 87^{\circ} \mathrm{W} 86^{\circ} \mathrm{W} 85^{\circ} \mathrm{W} 84^{\circ} \mathrm{W}$

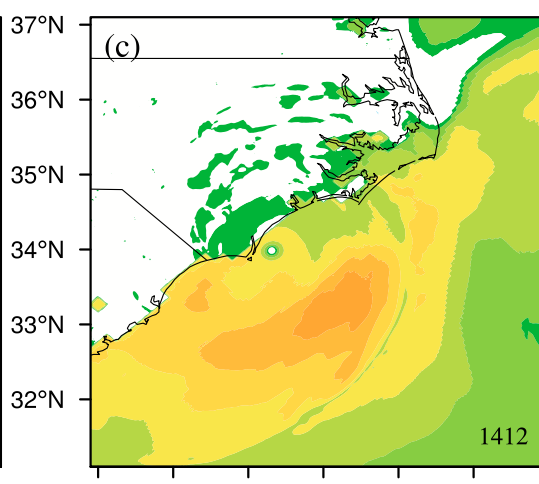

$80^{\circ} \mathrm{W} 79^{\circ} \mathrm{W} 78^{\circ} \mathrm{W} 77^{\circ} \mathrm{W} 76^{\circ} \mathrm{W} 75^{\circ} \mathrm{W}$

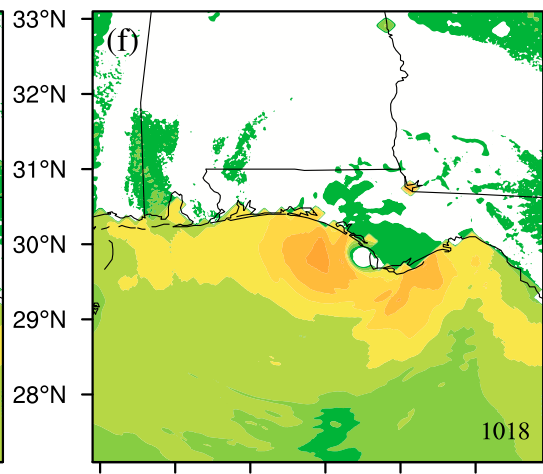

$89^{\circ} \mathrm{W} 88^{\circ} \mathrm{W} 87^{\circ} \mathrm{W} 86^{\circ} \mathrm{W} 85^{\circ} \mathrm{W} 84^{\circ} \mathrm{W}$

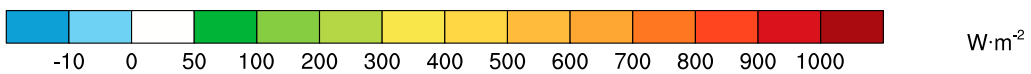

FIG. 8. Surface latent heat flux $\left(\mathrm{W} \mathrm{m}^{-2}\right.$ ) for Florence (1200 UTC 14 Sep 2018) and Michael (1800 UTC 10 Oct 2018) at landfall. The results of HRRR for (a) Florence and (d) Michael. The forecasts of (b) Exp. FCONV and (c) Exp. F06. The forecasts of (e) Exp. MCONV and (f) Exp. M06.

methods to reduce sampling errors by confining impacts of assimilated observations to nearby model grid points, should be furthered investigated. For convective-scale cases, a larger horizontal $(\sim 12-18 \mathrm{~km})$, but smaller vertical $(\sim 3 \mathrm{~km})$ localization is beneficial (Sobash and Stensrud 2013). However, for hurricane cases, localization correlation length may be different from what is suitable for convective cases. Exp. F04-05 and Exp. M04-05 focus on investigating the sensitivity of horizontal and vertical localization correlation length. With a horizontal localization correlation length of $400 \mathrm{~km}$ and a vertical localization correlation length of 10 grids, the southwestern underestimated region in Florence and the northwestern overestimated region in Michael both show further revisions.

\section{d. Weight of background error covariance}

The weighting value for ensemble background covariance is quite sensitive to the ensemble size. For large ensembles (50$100)$, a stronger weight to ensemble covariance is recommended (Gao and Stensrud 2014). In the current operational HWRF, the weight for ensemble covariance is 0.8. With the introduction of NEXRAD radial velocity assimilation into the current system, the sensitivity of different weights can be further tested. Exp. F05-07 and Exp. M05-07 (see Table 1) focus on investigating the sensitivity of background error covariance weight. For Florence, decreasing to a slight weighting value (e.g., from 0.8 to 0.7 ) to ensemble background covariance gains improvements in the southwestern underestimated region (greater than $38 \mathrm{~m} \mathrm{~s}^{-1}$ ). For Michael, the northwestern overestimated region gains a further revision. Exp. M06 provides a more reasonable value for the northwestern region, retaining the northeastern maximum wind information shown in Exp. MCONV.

With the various experimental designs above, a series of forecast tracks is presented in Fig. 4 for Florence (0600 UTC 14 September-0000 UTC 18 September 2018) Michael (1200 UTC 10 October-0000 UTC 12 October 2018), compared with available NHC best-track data. Since the experiment with conventional data assimilation already shows excellent performance on track forecast, only slight impacts (up to $5 \%$ of track error deductions in most of the cases) are gained by the additional assimilation of NEXRAD radial velocity. For the corresponded forecast intensity shown in Fig. 5, a similar tendency is captured by all NEXRAD experiments. For Hurricane Michael, there is a sudden intensification right at its landfall, which conventional and NEXRAD data assimilation experiments all miss. However, NEXRAD experiments amend intensity underestimation in the conventional experiments to some extent. Particularly, Exp. 

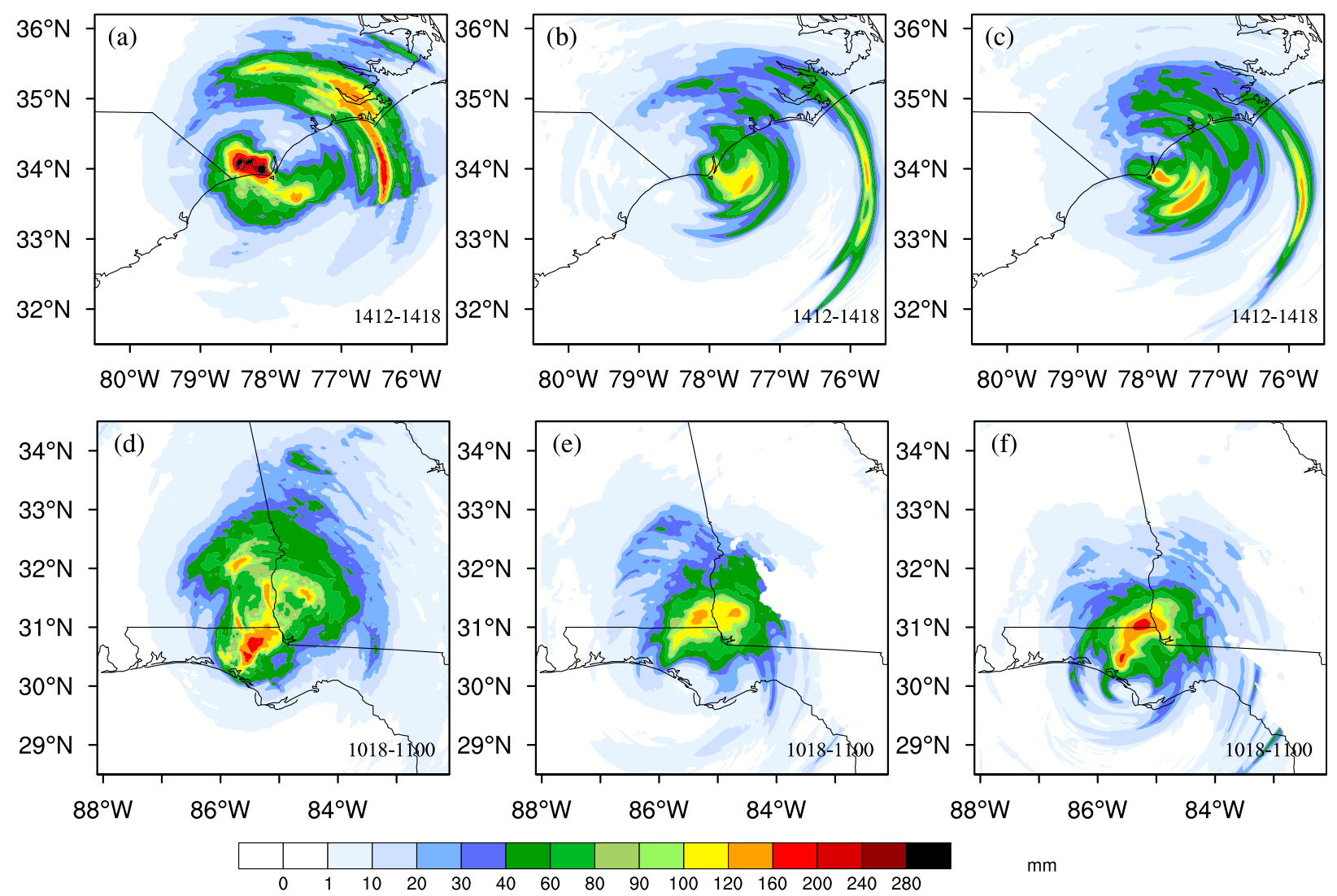

FIG. 9. Observed and forecast $6 \mathrm{~h}$ accumulated precipitation (mm) for Florence (1200-1800 UTC 14 Sep 2018) and Michael (18001100 UTC 10 Oct 2018) after landfall. The Stage IV products for (a) Florence and (d) Michael. The forecast precipitation of (b) Exp. FCONV and (c) Exp. F06. The forecast precipitation of (e) Exp. MCONV and (f) Exp. M06.

M06 achieves the best maximum wind estimation $6 \mathrm{~h}$ before Michael's landfall.

Overall, from the sensitivity of different parameters to the track and intensity forecasts, Exp. F06 and Exp. M06 both achieve the best performance among all the NEXRAD experiments.

\section{Discussion}

\section{a. Dynamical structures}

To investigate the impacts of the NEXRAD experiments on hurricane intensity and structure, the dynamical structure of horizontal and vertical cross-section wind fields is shown in Figs. 6 and 7 . The wind field of HRRR is utilized for verification. The asymmetric structure of the inner-core region plays an important role in the evolution, structure, and intensity change of hurricanes (e.g., Menelaou et al. 2013; Menelaou and Yau 2014). The horizontal forecast wind field in conventional experiments maintains a more symmetric structure, unlike the asymmetric structure in HRRR. After NEXRAD radial wind data are assimilated, the horizontal hurricane structure becomes looser. For the maximum wind region of the inner core (i.e., greater than $45 \mathrm{~m} \mathrm{~s}^{-1}$ for Florence and $50 \mathrm{~m} \mathrm{~s}^{-1}$ for Michael), Exp. F06 and Exp. M06 significantly revise its location and rebuild the asymmetric structure. In the vertical crosssection wind fields, the asymmetric convection structure of the left and right sides of the hurricane is better presented in the NEXRAD experiments. Exp. M06 revises the underestimation of the tangential wind field from 400 to $200 \mathrm{hPa}$ on the right side of the vortex, and the overestimation from 700 to $250 \mathrm{hPa}$ on the left side. Overall, the dynamical structure of hurricanes can be significantly revised by assimilating NEXRAD radial wind data.

\section{b. Thermodynamic field and precipitation}

Thermodynamic conditions play an essential role in hurricane evolution. Surface latent heat flux can depict the impact of underlying surface heating (shown in Fig. 8). For the surface latent heat flux field of Florence, an increase is apparent in the southeastern part of the hurricane vortex after NEXRAD radial wind data are assimilated. In the southwestern part, the high flux region shows a revised pattern similar to HRRR data in the NEXRAD assimilation experiments. For Michael, the high latent heat flux center is also located in the southwestern part of the vortex. After NEXRAD radial velocity are assimilated, although the magnitude of high centers is underestimated, the pattern in Exp. M06 is similar to HRRR data. Here, a more accurate heat of the underlying surface is provided for Michael, which contributes to intensity revision before landfall. 

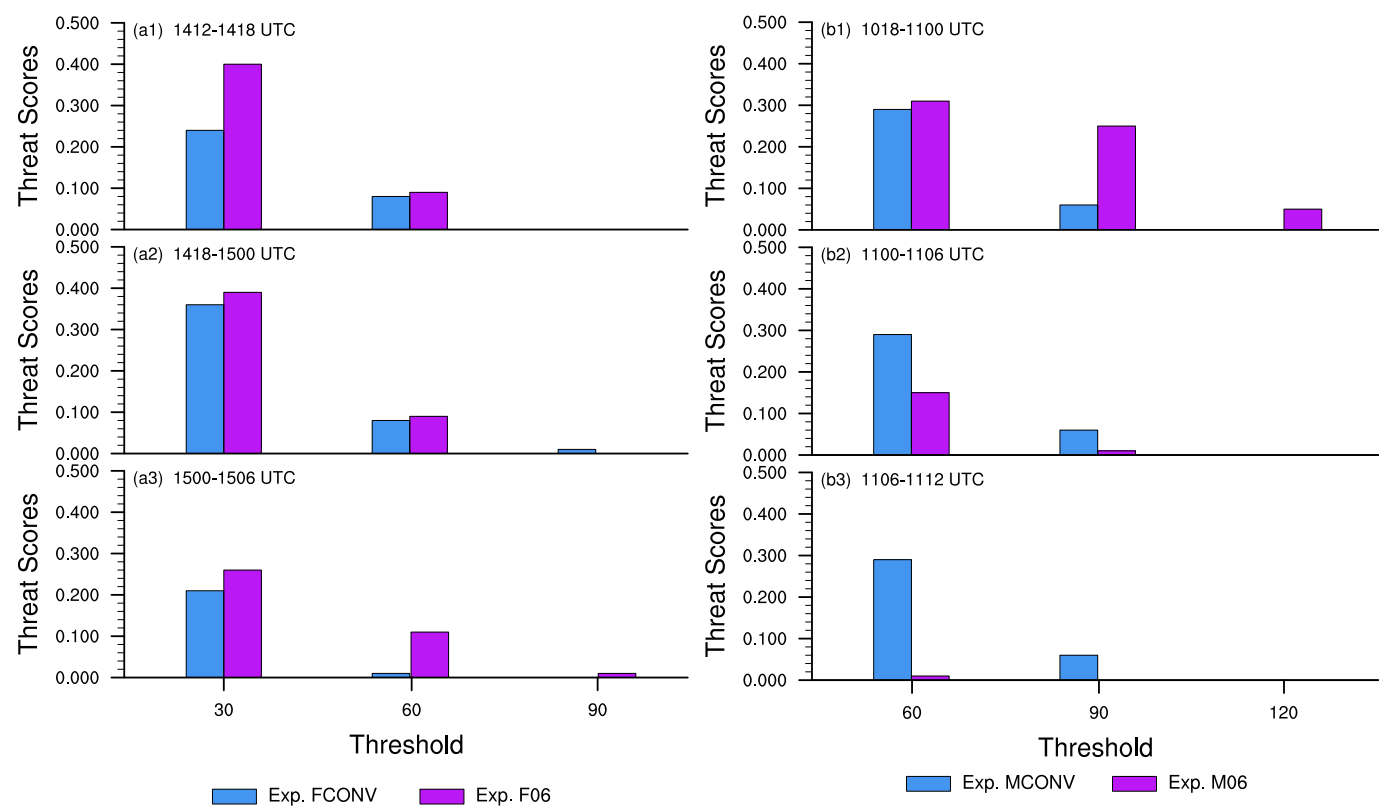

FIG. 10. The equitable threat scores (ETS) of quantitative precipitation forecasts (QPF) against Stage IV data for Hurricanes (left) Florence and (right) Michael after landfall. The calculation for Florence is from 1200 UTC 14 Sep to 0600 UTC 15 Sep 2018 at a 6-h interval, and for Michael is from 1800 UTC 10 Oct to 1200 UTC 11 Oct 2018 at a 6-h interval. Thresholds for Florence are 30, 60, and $90 \mathrm{~mm}$, and for Michael the thresholds are 60, 90, and $120 \mathrm{~mm}$.

Precipitation is another forecast focus for hurricane landfalls. Figure 9 illustrates the distribution of $6 \mathrm{~h}$ accumulated precipitation right after landfall. The NCEP Stage IV precipitation Data (Du 2011) are utilized to verify the forecast. For Florence, the conventional experiments underestimate the inner-core precipitation. Exp. F06 revises the underestimation at landfall. For Michael, Exp. M06 achieves similar precipitation (greater than $120 \mathrm{~mm}$ ) to the observations in the innercore region. Figure 10 evaluates a quantitative precipitation forecast with equitable threat scores against the Stage IV precipitation data for both Florence and Michael, improvements in the NEXRAD experiments for the quantitative precipitation forecast are maintained about $18 \mathrm{~h}$ after landfall.

\section{Summary}

HWRF has been in operation at NOAA NCEP for over a decade and has contributed a lot to hurricane forecasting and research. In the current operational platform, NEXRAD radial velocity data have not been employed as an assimilation data source until recently. The dpQC quality control algorithm employed in the MRMS system can remove nonhydrometeor echoes at a high computational efficiency. Based on data available from the real-time updated quality control algorithm, this study focuses on investigating the optimal operational configurations for NEXRAD radial velocity assimilation in HWRF and also examining its impacts on hurricane landfall. Two hurricane cases (i.e., Florence and Michael) in summer 2018 are used to conduct a series of experiments. Results are as follows.

- Compared with conventional experiments, the dynamical asymmetric structure of hurricanes can be better presented by assimilating radial wind data. This dynamical revision can further contribute to intensity forecasts, especially maximum wind forecasts before landfall.

- Improvements in quantitative precipitation forecasts are obvious, especially at high precipitation thresholds. These improvements can be maintained about $18 \mathrm{~h}$ after landfall with NEXRAD radial velocity assimilation.

- The optimal configurations for NEXRAD radial wind assimilation in HWRF are as follows, with the specific settings and version of the HWRF model used in this study. Maximum radial range for horizontal data coverage and radial range for superobservation boxes are 200 and $3 \mathrm{~km}$, respectively. Horizontal and vertical localization correlation lengths are $400 \mathrm{~km}$ and 10 grids. The weights applied to static background error covariance and ensemble covariance are 0.3 and 0.7 .

- Improvements in track forecasts are marginal in both hurricane cases with NEXRAD data assimilation when the shortrange track errors in control forecasts (with conventional data assimilation) have already been small. More case studies are needed to confirm the significant impacts of NEXRAD data assimilation on track forecasts.

Overall, studies with two hurricane cases have shown the great potential of implementing NEXRAD radial wind assimilation into the current HWRF operational platform. Specifically, this study identified important sensitivity parameters for the operational implementation of NEXRAD data assimilation with the GSI system. With the expected upgrades of the HWRF system in the operational environment, more cases and more detailed evaluation are needed to investigate and eventually make operational improvements in hurricane forecasts. 
Acknowledgments. We are grateful to Drs. L. Bi and A. Mehra at NCEP/EMC, Dr. M. Hu at NOAA/ESRL, and H. Reeves at NOAA/NSSL for helpful discussion and useful comments. This study is supported by NOAA Award NA19OAR4590239 and NSF Grant OAC-2004658. The computational resources and support from NOAA Jet supercomputer system (for Dr. Z. Pu) and NCAR CISL Cheyenne supercomputer system are appreciated. All inputs NEXRAD data come from the NCEP operational archive. The NCAR Developmental Testbed Center (https://dtcenter.org) is also acknowledged for supporting the HWRF and GSI software. NCEP ADP data can be obtained from the website (https://rda.ucar.edu/datasets/ds337.0/).

\section{REFERENCES}

Alpert, J. C., and V. K. Kumar, 2007: Radial wind super-obs from the WSR-88D radars in the NCEP operational assimilation system. Mon. Wea. Rev., 135, 1090-1109, https://doi.org/ 10.1175/MWR3324.1.

Ansari, S., and Coauthors, 2018: Unlocking the potential of NEXRAD data through NOAA's big data partnership. Bull. Amer. Meteor. Soc., 99, 189-204, https://doi.org/ 10.1175/BAMS-D-16-0021.1.

Biswas, M. K., and Coauthors, 2018: Hurricane Weather Research and Forecasting (HWRF) Model: 2018 scientific documentation. Developmental Testbed Center, 112 pp., https:/dtcenter.org/ sites/default/files/community-code/hwrf/docs/scientific_documents/ HWRFv4.0a_ScientificDoc.pdf.

Bytheway, J. L., C. D. Kummerow, and C. Alexander, 2017: A features-based assessment of the evolution of warm season precipitation forecasts from the HRRR model over three years of development. Wea. Forecasting, 32, 1841-1856, https://doi.org/10.1175/WAF-D-17-0050.1.

Chen, F., and J. Dudhia, 2001: Coupling an advanced land surfacehydrology model with the Penn State-NCAR MM5 modeling system. Part I: Model description and implementation. Mon. Wea. Rev., 129, 569-585, https://doi.org/10.1175/1520-0493(2001) 129<0569:CAALSH $>2.0 . \mathrm{CO} ; 2$.

Du, J., 2011: GCIP/EOP surface: Precipitation NCEP/EMC 4KM Gridded Data (GRIB) Stage IV Data, version 1.0. UCAR/ NCAR-Earth Observing Laboratory, accessed 5 February 2021, https://doi.org/10.5065/D6PG1QDD.

Ferrier, B. S., Y. Jin, Y. Lin, T. Black, E. Rogers, and G. DiMego, 2002: Implementation of a new grid-scale cloud and precipitation scheme in the NCEP Eta model. 19th Conf. on Weather Analysis and Forecasting/15th Conf. on Numerical Weather Prediction, San Antonio, TX, Amer. Meteor. Soc., 10.1, https://ams.confex.com/ams/SLS_WAF_NWP/techprogram/ paper_47241.htm.

Gao, J.-D., and D. J. Stensrud, 2012: Assimilation of reflectivity data in a convective-scale, cycled 3DVAR framework with hydrometeor classification. J. Atmos. Sci., 69, 1054-1065, https://doi.org/10.1175/JAS-D-11-0162.1.

$\longrightarrow$, and — 2014: Some observing system simulation experiments with a hybrid 3DEnVAR system for storm-scale radar data assimilation. Mon. Wea. Rev., 142, 3326-3346, https:// doi.org/10.1175/MWR-D-14-00025.1.

Han, J., and H.-L. Pan, 2011: Revision of convection and vertical diffusion schemes in the NCEP Global Forecast System. Wea. Forecasting, 26, 520-533, https://doi.org/10.1175/WAF-D-1005038.1.

- M. Witek, J. Teixeira, R. Sun, H.-L. Pan, J. K. Fletcher, and C. S. Bretherton, 2016: Implementation in the NCEP GFS of a hybrid Eddy-Diffusivity Mass-Flux (EDMF) boundary layer parameterization with dissipative heating and modified stable boundary layer mixing. Wea. Forecasting, 31, 341-352, https:// doi.org/10.1175/WAF-D-15-0053.1.

Hong, S.-Y., and H.-L. Pan, 1998: Convective trigger function for a mass flux cumulus parameterization scheme. Mon. Wea. Rev., 126, 2621-2639, https://doi.org/10.1175/15200493(1998)126<2621:IOPCSF>2.0.CO;2.

Hu, J.-X., D. Rosenfeld, A. Ryzhkov, and P.-F. Zhang, 2020: Synergetic use of the WSR-88D radars, GOES-R satellites, and lightning networks to study microphysical characteristics of hurricanes. J. Appl. Meteor. Climatol., 59, 1051-1068, https://doi.org/10.1175/JAMC-D-19-0122.1.

Hu, M., G. Ge, C. Zhou, D. Stark, H. Shao, K. Newman, J. Beck, and X. Zhang, 2018: Grid-point Statistical Interpolation (GSI) user's guide version 3.7. Developmental Testbed Center, 149 pp., https://dtcenter.org/community-code/gridpoint-statisticalinterpolation-gsi/documentation.

Iacono, M. J., J. S. Delamere, E. J. Mlawer, M. W. Shephard, S. A. Clough, and W. D. Collins, 2008: Radiative forcing by longlived greenhouse gases: Calculations with the AER radiative transfer models. J. Geophys. Res., 113, D13103, https://doi.org/ 10.1029/2008JD009944.

Janjić, Z. I., R. Gall, and M. E. Pyle, 2010: Scientific documentation for the NMM solver. NCAR Tech. Note NCAR/TN-477+STR, 53 pp., https://doi.org/10.5065/D6MW2F3Z.

Jung, Y., M. Xue, and G. Zhang, 2010: Simulations of polarimetric radar signatures of a supercell storm using a two-moment bulk microphysics scheme. J. Appl. Meteor. Climatol., 49, 146-163, https://doi.org/10.1175/2009JAMC2178.1.

Lai, A.-W., J.-D. Gao, S. E. Koch, Y.-H. Wang, S.-J. Pan, A. O. Fierro, C.-G. Cui, and J.-Z. Min, 2019: Assimilation of radar radial velocity, reflectivity, and pseudo-water vapor for convective-scale NWP in a variational framework. Mon. Wea. Rev., 147, 28772900, https://doi.org/10.1175/MWR-D-18-0403.1.

Laroche, K. T., and T. J. Lang, 2017: Observations of ash, ice, and lightning within pyrocumulus clouds using polarimetric NEXRAD radars and the National Lightning Detection Network. Mon. Wea. Rev., 145, 4899-4910, https://doi.org/10.1175/MWR-D-17-0253.1.

Li, Z., Z. Pu, J. Sun, and W.-C. Lee, 2014: Impacts of 4D-VAR assimilation of airborne Doppler radar observations on numerical simulations of the genesis of Typhoon Nuri (2008). J. Appl. Meteor. Climatol., 53, 2325-2343, https://doi.org/ 10.1175/JAMC-D-14-0046.1.

Lippi, D. E., J. R. Carley, and D. T. Kleist, 2019: Improvements to the assimilation of Doppler radial winds for convectionpermitting forecasts of a heavy rain event. Mon. Wea. Rev., 147, 3609-3632, https://doi.org/10.1175/MWR-D-18-0411.1.

Menelaou, K., and M. K. Yau, 2014: On the role of asymmetric convective bursts to the problem of hurricane intensification: Radiation of vortex Rossby waves and wave-mean flow interactions. J. Atmos. Sci., 71, 2057-2077, https://doi.org/10.1175/JAS-D-13-0343.1.

,-- , and Y. Martinez, 2013: Impacts of asymmetric dynamical processes on the structure and intensity change of two-dimensional hurricane-like annular vortices. J. Atmos. Sci., 70, 559-582, https://doi.org/10.1175/JAS-D-12-0192.1.

Mitchell, K., 2005: The community Noah Land Surface Model (LSM). NCAR, 26 pp., https://ral.ucar.edu/sites/default/files/ public/product-tool/unified-noah-lsm/Noah_LSM_USERGUIDE_ 2.7.1.pdf.

Pan, H.-L., 2003: The GFS Atmospheric Model. NCEP Office Note 442, 14 pp. [Available from NCEP, 5200 Auth Road, Washington, DC 20233]. 
and J. Wu, 1995: Implementing a mass flux convection parameterization package for the NMC medium-range forecast model. NMC Office Note 409, 40 pp. [Available from NCEP, 5200 Auth Road, Washington, DC 20233.]

Posselt, D. J., X. Li, S. A. Tushaus, and J. R. Mecikalski, 2015: Assimilation of dual-polarization radar observations in mixed- and ice- phase regions of convective storms: Information content and forward model errors. Mon. Wea. Rev., 143, 26112636, https://doi.org/10.1175/MWR-D-14-00347.1.

Pu, Z., X. Li, and J. Sun, 2009: Impact of airborne Doppler radar data assimilation on the numerical simulation of intensity changes of Hurricane Dennis near a landfall. J. Atmos. Sci., 66, 3351-3365, https://doi.org/10.1175/2009JAS3121.1.

Rappaport, E. N., and Coauthors, 2009: Advances and challenges at the National Hurricane Center. Wea. Forecasting, 24, 395419, https://doi.org/10.1175/2008WAF2222128.1.

Snyder, C., and F. Zhang, 2003: Assimilation of simulated Doppler radar observations with an ensemble Kalman filter. Mon. Wea. Rev., 131, 1663-1677, https://doi.org/10.1175//2555.1.

Sobash, R. A., and D. J. Stensrud, 2013: The impact of covariance localization for radar data on EnKF analyses of a developing MCS: Observing system simulation experiments. Mon. Wea. Rev., 141, 3691-3709, https://doi.org/10.1175/MWR-D-12-00203.1.

Sun, J., 2005: Initialization and numerical forecasting of a supercell storm observed during STEPS. Mon. Wea. Rev., 133, 793-813, https://doi.org/10.1175/MWR2887.1.

_ , and N. A. Crook, 1997: Dynamical and microphysical retrieval from Doppler radar observations using a cloud model and its adjoint. Part I: Model development and simulated data experiments. J. Atmos. Sci., 54, 1642-1661, https://doi.org/ 10.1175/1520-0469(1997)054<1642:DAMRFD>2.0.CO;2.

Tang, L., J. Zhang, C. Langston, J. Karuse, K. Howard, and V. Lakshmanan, 2014: A physically based precipitationnonprecipitation radar echo classifier using polarimetric and environmental data in a real-time national system. Wea. Forecasting, 29, 1106-1119, https://doi.org/10.1175/WAF-D-13-00072.1.

Tong, M., and M. Xue, 2005: Ensemble Kalman filter assimilation of Doppler radar data with a compressible nonhydrostatic model: OSS experiments. Mon. Wea. Rev., 133, 1789-1807, https://doi.org/10.1175/MWR2898.1.
Wang, X., 2010: Incorporating ensemble covariance in the Gridpoint Statistical Interpolation (GSI) variational minimization: A mathematical framework. Mon. Wea. Rev., 138, 2990-2995, https://doi.org/10.1175/2010MWR3245.1.

Xiao, Q., Y.-H. Kuo, J. Sun, W.-C. Lee, E. Lim, Y.-R. Guo, and D. M. Barker, 2005: Assimilation of Doppler radar observations with a regional 3DVAR system: Impact of Doppler velocities on forecasts of a heavy rainfall case. J. Appl. Meteor., 44, 768-788, https://doi.org/10.1175/ JAM2248.1.

Yablonsky, R. M., I. Ginis, and B. Thomas, 2015: Ocean modeling with flexible initialization for improved coupled tropical cyclone-ocean model prediction. Environ. Modell. Software, 67, 26-30, https://doi.org/10.1016/j.envsoft.2015.01.003.

Yue, H., and M. Cebremichael, 2020: Evaluation of high-resolution rapid refresh (HRRR) forecasts for extreme precipitation. Environ. Res. Commun., 2, 065004, https://doi.org/10.1088/ 2515-7620/ab9002.

Zhang, J., and Coauthors, 2011: National Mosaic and Multi-Sensor QPE (NMQ) system: Description, results, and future plans. Bull. Amer. Meteor. Soc., 92, 1321-1338, https://doi.org/ 10.1175/2011BAMS-D-11-00047.1.

—_, and Coauthors, 2016: Multi-Radar Multi-Sensor (MRMS) quantitative precipitation estimation: Initial operating capabilities. Bull. Amer. Meteor. Soc., 97, 621-638, https://doi.org/ 10.1175/BAMS-D-14-00174.1.

Zhang, L., Z. Pu, W.-C. Lee, and Q. Zhao, 2012: The influence of airborne Doppler radar data quality control on numerical simulations of a tropical cyclone. Wea. Forecasting, 27, 231239, https://doi.org/10.1175/WAF-D-11-00028.1.

Zhang, S.-X., and Z. Pu, 2019: Numerical simulation of rapid weakening of Hurricane Joaquin with assimilation of highdefinition sounding system dropsondes during the Tropical Cyclone Intensity Experiment: Comparison of three- and four-dimensional ensemble-variational data assimilation. Wea. Forecasting, 34, 521-538, https://doi.org/10.1175/WAFD-18-0151.1.

Zhao, Q., and Y. Jin, 2008: High-resolution radar data assimilation for Hurricane Isabel (2003) at landfall. Bull. Amer. Meteor. Soc., 89, 1355-1372, https://doi.org/10.1175/2008BAMS2562.1. 